\title{
Control sideband generation for dual-recycled laser interferometric gravitational wave detectors
}

\author{
B W Barr 1 , O Miyakawa ${ }^{2}$, S Kawamura ${ }^{3}$, A J Weinstein ${ }^{2}$, R Ward ${ }^{2}$, \\ S Vass ${ }^{2}$ and K A Strain ${ }^{1}$ \\ ${ }^{1}$ Department of Physics and Astronomy, University of Glasgow, Glasgow, G12 8QQ, UK \\ ${ }^{2}$ LIGO Laboratory, California Institute of Technology, Pasadena, CA 91125, USA \\ ${ }^{3}$ National Astronomical Observatory of Japan, Tokyo 1818588 , Japan \\ E-mail: b.barr@physics.gla.ac.uk
}

Received 19 June 2006, in final form 26 July 2006

Published 22 August 2006

Online at stacks.iop.org/CQG/23/5661

\begin{abstract}
We present a discussion of the problems associated with generation of multiple control sidebands for length sensing and control of dual-recycled, cavity-enhanced Michelson interferometers and the motivation behind more complicated sideband generation methods. We focus on the Mach-Zehnder interferometer as a topological solution to the problem and present results from tests carried out at the Caltech $40 \mathrm{~m}$ prototype gravitational wave detector. The consequences for sensing and control for advanced interferometry are discussed, as are the implications for future interferometers such as Advanced LIGO.
\end{abstract}

PACS numbers: 42.60.-v, 42.60.Fc

There are several large scale projects around the world building and operating laser interferometric gravitational-wave detectors including LIGO [1] (USA), GEO600 [2] (UK/Germany), VIRGO [3] (France/Italy) and TAMA [4] (Japan). These detectors are based around enhanced Michelson interferometer optical topologies and operate by detecting fluctuations in optical phase produced by the relative displacement of mirrors responding to gravitational radiation.

These first generation detectors are expected to open up the field of gravitational wave astronomy with a detection event rate of up to a few events per year. The second generation of detectors is being developed to improve the peak gravitational wave detection sensitivity by more than an order of magnitude and improve the expected event rate by several orders of magnitude [5]. These advanced detectors will incorporate several enhancements over the basic Michelson design and will have the general form shown in figure 1. The interferometer has a specific operating condition requiring the relative lengths between optics to be sensed and controlled. The proposed sensing and control scheme for the Advanced LIGO [6] detectors is currently being investigated using the Caltech $40 \mathrm{~m}$ prototype interferometer. 


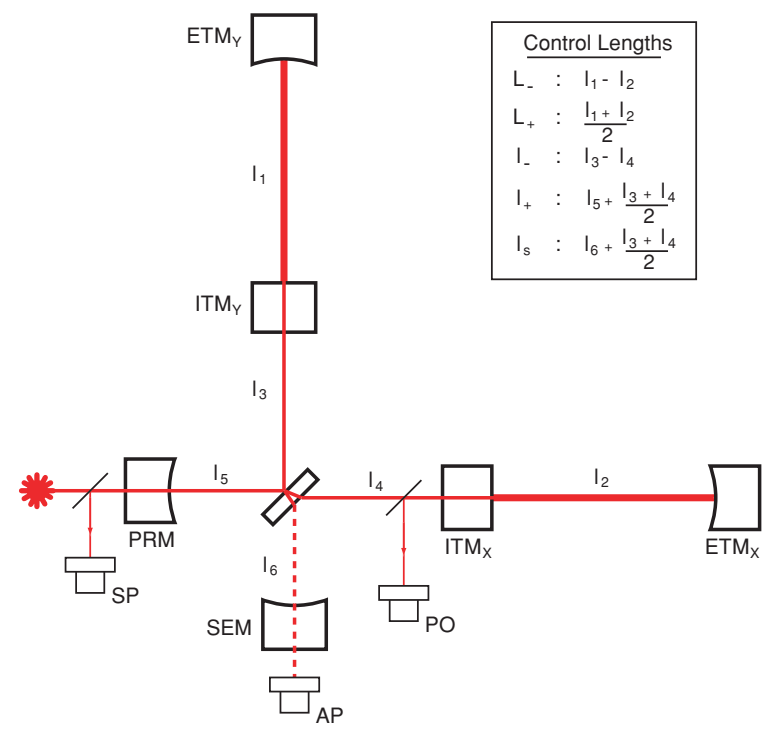

Figure 1. General form of the Advanced LIGO detectors and the $40 \mathrm{~m}$ prototype interferometer. The logical control lengths (or degrees of freedom) $L_{-}, L_{+}, l_{+}, l_{-}$and $l_{s}$ are shown, and three possible points for signal detection are identified as SP (symmetric port), AP (antisymmetric port) and PO (pick-off port).

The sensing and control scheme being considered for this system has been discussed in detail [7] and is an extrapolation from the well-known Pound-Drever-Hall reflection locking scheme [8] where phase modulation sidebands are imposed on the laser light (carrier). After interaction with the interferometer the light is detected at all three detection ports and the signals are demodulated to give a bipolar control signal for each degree of freedom.

With five degrees of freedom and only three available detection points the sensing and control scheme being investigated requires the use of two sets of phase-modulated control sidebands (at frequencies $f_{1}$ and $f_{2}$ ). In the $40 \mathrm{~m}$ system $f_{2}=5 f_{1}$, where $f_{1}=33.033 \mathrm{MHz}$. For Advanced LIGO the proposed values of $f_{1}$ and $f_{2}$ are $9 \mathrm{MHz}$ and $180 \mathrm{MHz}$, respectively, where these frequencies depend on the interferometer topology and will give improved decoupling between sensing signals than is possible with the $40 \mathrm{~m}$ system. The sensing signals chosen to control the central part of the interferometer (lengths $l_{+}, l_{-}$and $l_{s}$ ) are derived from the beat signals $\left(f_{1} \pm f_{2}\right)$ between the sidebands (at ports SP, AP and PO respectively). The arm cavity sensing signals are derived from demodulation of the beat between one of the sidebands and the carrier using $f_{2}$ at AP to sense $L_{-}$(the gravitational wave detection length) and $f_{1}$ at SP to sense $L_{+}$. One of the primary advantages of this double demodulation sensing method is the decoupling of the central degrees of freedom from the arm cavities.

At the desired operating point the arm cavities $\left(L_{-}\right.$and $\left.L_{+}\right)$and power recycling cavity $\left(l_{+}\right)$must be held on resonance with the incident carrier light, the output port must be held to a dark carrier fringe $\left(l_{-}\right)$and the signal recycling cavity $\left(l_{s}\right)$ must be held to a specific resonance condition determined by the desired frequency response [7]. The control sidebands also resonate within the system to allow generation of length sensing signals. In the $40 \mathrm{~m}$ system, both sets of sidebands (at $\pm f_{1}$ and $\pm f_{2}$ ) are resonant in the power recycling cavity, while only one sideband $\left(\right.$ at $+f_{2}$ ) is resonant in the detuned signal recycling cavity. This unbalanced sideband system means that, while the interferometer is held to the operating 

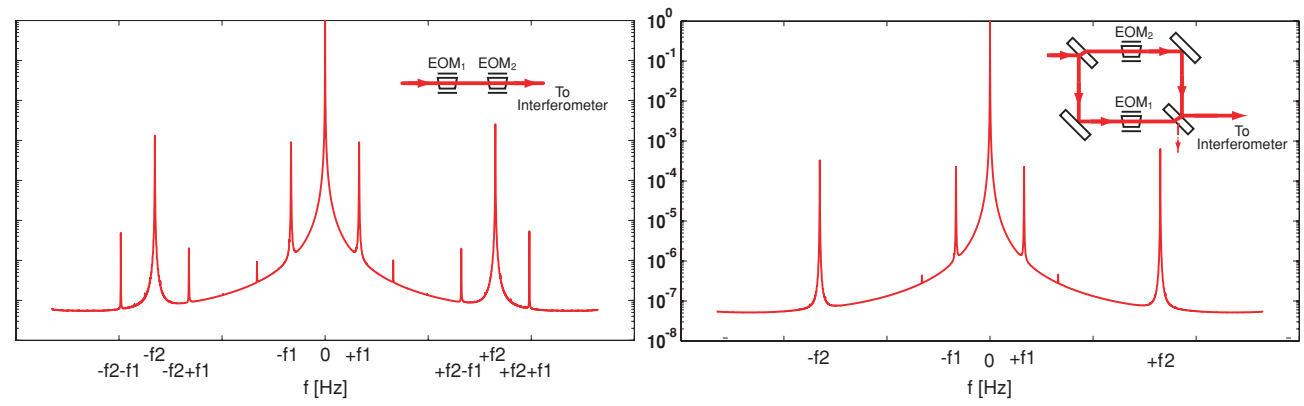

Figure 2. The left-hand diagram shows the frequency components of the light with sidebands on sidebands as generated by a conventional series modulator configuration. The right-hand side shows the required frequency components for a viable double demodulation sensing and control scheme as generated using a parallel modulator (Mach-Zehnder) configuration. The inset diagrams are more clearly displayed in figure 3 .

point, different sidebands have different responses to the motion of different mirrors. Through careful selection of demodulation phase for each double demodulation signal, the coupling between the length sensing signals for $l_{+}, l_{-}$and $l_{s}$ can be minimized. Since the central part of the interferometer is already decoupled from the arm cavities the sensing scheme for the system is optimally decoupled at the operating point, resulting in a roughly diagonal control matrix.

Double demodulation presents an additional difficulty with regard to the sideband generation requirements for advanced interferometer control. Generation via phase modulation of one set of sidebands of amplitude $\beta$ at frequency $f_{2}$ on top of another set of amplitude $\alpha$ at frequency $f_{1}$ will impose additional sets of sidebands offset from the carrier (amplitude 1) at frequencies $f_{1} \pm f_{2}$ with amplitude $\alpha \beta$. This effect can be seen in figure 2 and has been termed 'sidebands on sidebands'. A signal produced by the beat between sidebands on sidebands and the carrier will result in a signal $(\alpha \beta \times 1=\alpha \beta)$ of the same magnitude as one derived from the beat between sidebands $(\alpha \times \beta=\alpha \beta)$. Modelling of this system indicates that due to the high finesse of the arm cavities, the arm cavity degrees of freedom will not only be strongly coupled with the central part of the interferometer, but will actually be the dominant elements of the sensing signals for the central degrees of freedom. A sideband generation scheme must be used which does not result in sidebands of sidebands being present on the light. One such scheme is to use parallel modulators in a Mach-Zehnder interferometer configuration (see right-hand configuration in figure 2).

The Mach-Zehnder configuration solves the problem of sidebands on sidebands, but for the purposes of high sensitivity interferometry the beams in each arm must be recombined in phase. Residual deviations from the desired recombination phase will introduce noise into the system and limit the interferometer detection sensitivity.

These residual motions were extensively modelled with respect to the effect on the $L_{-}$ signal using the FINESSE interferometer simulation program [9]. The system was modelled with differences between the ITM transmissions of up to $\pm 10 \%$. This represents the maximum imbalance expected in the $40 \mathrm{~m}$ system. A more balanced system will have less susceptibility to noise coupling to the $L_{-}$output signal.

Noise can be introduced in two main ways, namely common mode and differential mode fluctuations of the two Mach-Zehnder arm lengths. Differential arm length fluctuations couple directly to the $L_{-}$signal by introducing fluctuations in the relative phase between the carrier 

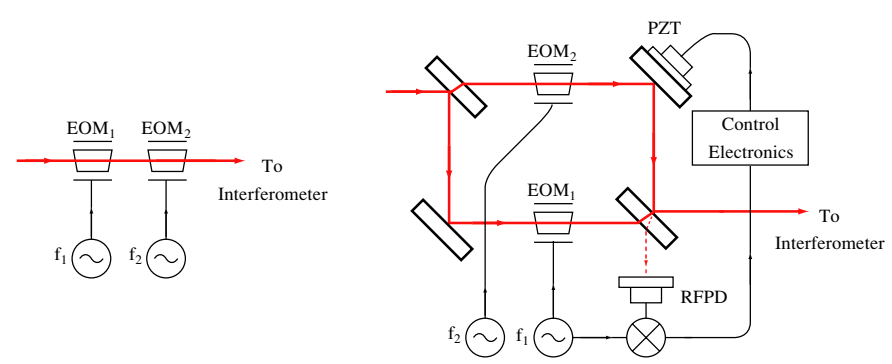

Figure 3. Left: conventional modulator configuration. Right: Mach-Zehnder modulation configuration and control loop. The signal at the RF photodiode (RFPD) is demodulated to provide a length sensing signal. This signal is fed back to the PZT in the $f_{2}$ arm to control the relative arm lengths, keeping the PD port dark for the carrier light.

and the sidebands at $f_{1}$ and $f_{2}$. These can only be reduced by suppressing the fluctuations in the Mach-Zehnder differential arm length. Modelling of the system [10] gives the coupling factor for differential Mach-Zehnder arm motion to $L_{-}$motion as around $10^{-6}$ for a $\pm 10 \%$ difference between ITMs. In contrast, common mode arm length fluctuations couple indirectly through other control systems - in this case the strongest indirect coupling mechanism is via the laser frequency stabilization servo loop. Modelling gives the coupling factor for common Mach-Zehnder arm motion to $L_{-}$motion as around $10^{-7}$ for a $\pm 10 \%$ difference between ITMs. The dominant mechanism for noise coupling is therefore direct coupling of differential Mach-Zehnder arm length fluctuations to the $L_{-}$output signal. These noise couplings will also be present at comparable levels in the Advanced LIGO detectors, and quantitative estimates of the required performance of the modulation system for Advanced LIGO are in progress.

The Mach-Zehnder interferometer as built at the $40 \mathrm{~m}$ lab is shown in figure 3 . Prestabilized laser light enters the Mach-Zehnder interferometer, is split such that half the incident power passes through each modulator and the beam is recombined in phase. In this state, half the sideband power passes to the Mach-Zehnder dark port and the modulator-driving signal must be increased to provide the modulation index of 0.1 after recombination required by the $40 \mathrm{~m}$ system. A servo loop is used to hold the relative arm lengths of the Mach-Zehnder constant and thus maintain the phase relationship between the sets of sidebands. The system uses one set of sidebands $\left(f_{1}\right)$, detected at the Mach-Zehnder dark port and demodulated, to derive a control signal which subsequently feeds back to a piezo-electric transducer (PZT) bonded to one of the mirrors. The unity gain frequency of the loop is $\sim 11 \mathrm{kHz}$.

Fluctuations on the signal are calibrated to give the residual change in relative arm length (see figure 4). The residual noise of the Mach-Zehnder can be seen to rise above $10^{-12} \mathrm{~m} \mathrm{~Hz}^{-1 / 2}$ in the several hundred $\mathrm{Hz}$ to $1 \mathrm{kHz}$ band. This region is contaminated by noise due to mechanical and electronic resonances associated with the optics, sensor photodiode, PZT actuator and the servo system. With a $10 \%$ imbalance in ITM transmissivity the direct coupling mechanism will result in a detector sensitivity limit of around $10^{-18} \mathrm{~m} \mathrm{~Hz}^{-1 / 2}$ from the $L_{-}$sensing output. This is around two orders of magnitude higher than the design specification for the prototype system.

The performance of the Mach-Zehnder servo loop could be easily improved by increasing the gain; but in the configuration shown, gain is limited by intrinsic resonances of the PZT. The servo bandwidth could be increased by inserting a phase correcting electro-optic modulator in one of the Mach-Zehnder arms to feed back at higher frequencies and allowing the gain to be increased in the region of interest. Using this method the residual length fluctuations could be 


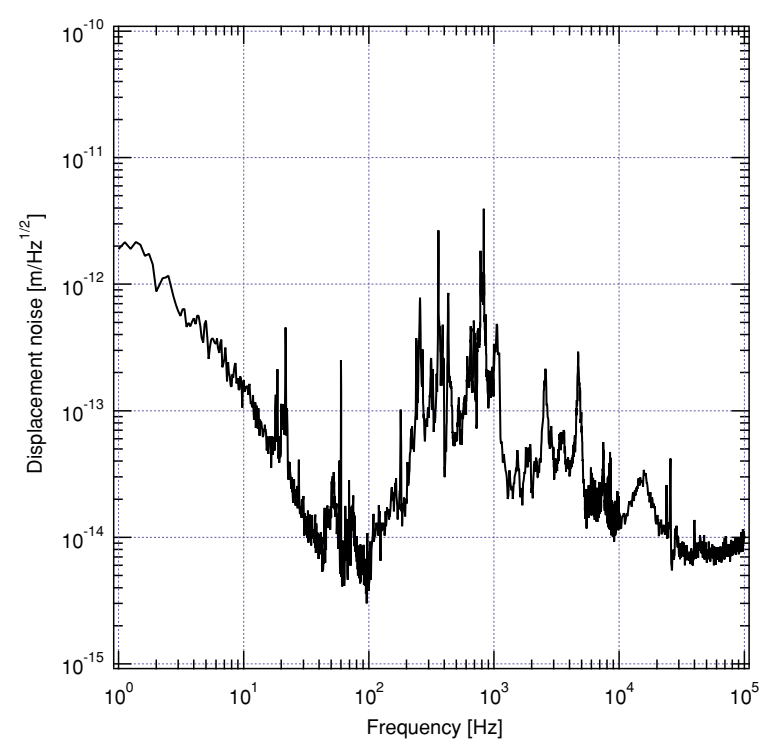

Figure 4. Mach-Zehnder residual noise calibrated as fluctuations in the relative arm length.

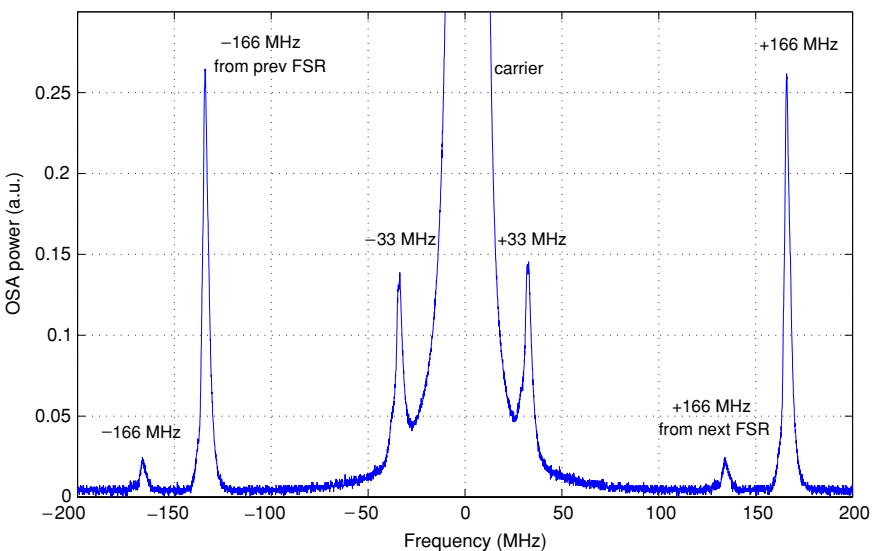

Figure 5. Frequency components of the modulated laser light measured on reflection from the interferometric system. There are no additional frequency components observed at the beat between the sideband frequencies. The unbalanced sidebands are the result of the design reflectivity of the detuned interferometer.

reduced by several orders of magnitude. It is expected that this will be sufficient for meeting the anticipated requirements on the modulation system for the Advanced LIGO detectors.

Using the Mach-Zehnder parallel modulation scheme, sidebands have been generated without sidebands on sidebands (shown in figure 5). The resulting length sensing signals have been used to lock the prototype interferometric system in a full Advanced LIGO configuration [11]. The work presented here demonstrates that, given a suitably high gain servo loop, the parallel modulation scheme is a viable option for the generation of control sidebands in full scale advanced interferometric gravitational wave detectors. 


\section{Acknowledgments}

This work is supported by the Particle Physics and Astronomy Research Council and the University of Glasgow in the UK and from the National Science Foundation cooperative agreement PHY0107417. This document has been assigned to LIGO Laboratory document number LIGO-P060022-02-Z. We thank the many members of the LIGO Laboratory, the LIGO Scientific Collaboration and many valuable visitors to the 40 meter lab for their invaluable contributions.

\section{References}

[1] Sigg D et al 2002 Class. Quantum Grav. 19 1429-35

[2] Wilke B et al 2002 Class. Quantum Grav. 19 1377-87

[3] Acernese F et al 2002 Class. Quantum Grav. 19 1421-8

[4] Ando M et al 2002 Class. Quantum Grav. 19 1409-19

[5] Bishop N (ed) 2002 An overview of gravitational-wave sources Proc. GR16 Conf. on General Relativity and Gravitation (Singapore: World Scientific)

[6] Gustafson E, Shoemaker D, Strain K and Weiss R 1999 LSC White Paper on Detector Research and Development, LIGO Document T990080-00-D

[7] Strain K A et al 2003 Appl. Opt. 42 1244-56

[8] Drever R W P, Hall J L, Kawalski F W, Hough J, Ford G M, Munley A J and Ward H 1981 Appl. Phys. B 31 97-105

[9] Freise A FINESSE_Frequency Domain Interferometer Simulation Software available from http://www.rzg.mpg.de/adf/

[10] Kawamura S and Miyakawa O 2004 LIGO Internal Note available at http://www.ligo.caltech.edu/ cit40m/Docs/T040166-00.pdf

[11] Miyakawa O et al 2006 Measurement of optical response of a detuned resonant sideband extraction gravitational wave detector Phys. Rev. D 74022001 (Preprint gr-qc/0604078) 